\title{
SINGLE-WALLED CARBON NANOTUBE MICROPATTERNS AND CANTILEVER ARRAY FABRICATED WITH ELECTROSTATIC LAYER-BY-LAYER NANO SELF-ASSEMBLY AND LITHOGRAPHY
}

\author{
Wei Xue and Tianhong Cui* \\ Department of Mechanical Engineering, University of Minnesota \\ 111 Church Street SE, Minneapolis, MN 55455, USA \\ Tel: 612-626-1636 Fax: 612-625-6069 E-mail: tcui@me.umn.edu
}

\begin{abstract}
A simple and effective approach is presented for patterning single-walled carbon nanotube (SWNT) microstructures by combining electrostatic layer-by-layer (LbL) nano self-assembly, lithography, and lift-off. This technique provides a simple, lowcost, and low-temperature fabrication method with a short processing time. SWNT micropatterns with feature sizes as small as $5 \mu \mathrm{m}$ were fabricated and characterized. The thickness of a (PDDA/SWNT) bi-layer was measured approximately as $76 \AA$. To investigate the potential applications of SWNTs, magnetic cantilever arrays consist of SWNTs, iron oxide $\left(\mathrm{Fe}_{2} \mathrm{O}_{3}\right)$ nanoparticles, and polyelectrolytes were developed. Due to the outstanding mechanical properties of the SWNTs, the fabricated cantilever arrays are strong and flexible. The cantilever arrays can be used in applications such as biosensors and microvalves.
\end{abstract}

\section{INTRODUCTION}

Since the discovery in 1991, carbon nanotubes (CNTs) have been investigated extensively due to their unique structural, electrical, chemical, and mechanical properties [1]. CNTs are promising materials in nanotechnology and can be used in a wide range of applications including chemical sensors, biosensors, stress/strain sensors, scanning probes, field emission displays, nanoelectronics devices, and nanoelectromechanical systems (NEMS) [2-5]. In their natural forms, CNTs are highly sensitive to molecules such as $\mathrm{NO}_{2}$ and $\mathrm{NH}_{3}$ [2]. With rational chemical and/or physical modification, CNTs are capable of detecting more types of molecules (e.g., $\mathrm{H}_{2}, \mathrm{CO}$, glucose, and DNA) [6]. The controllable deposition of CNTs, either individually or as a bulk material, is an essential step in building practical devices.

Current deposition approaches include chemical vapor deposition (CVD), selective electrophoresis deposition, Langmuir-Blodgett method, and nano-manipulation using atomic force microscope tips [7-8]. However, commercialization of CNTbased devices demands faster, less expensive, and more convenient deposition techniques. Electrostatic layer-by-layer (LbL) nano self-assembly was introduced to produce films in micro- and nanoscale. LbL nano self-assembly is an effective and economic approach to build well-organized multilayers in nanometer scale. LbL self-assembled thin films can be deposited on the surface of almost any materials with any topography [9]. With this technique, SWNT thin films have been fabricated and investigated by several groups. Compared with other SWNT/polymer matrices, LbL self-assembled SWNT thin films show enhanced mechanical properties [10]. In addition, the thin films fabricated with LbL self-assembly usually retain the electrochemical catalytic activities of the CNTs [11].

In this paper, we report a simple, effective, and versatile technique to fabricate SWNT microstructures through a combination of electrostatic LbL nano self-assembly and conventional lithography. This technique provides a simple, lowcost, and low-temperature fabrication method with a short processing time. The SWNT films and micropatterns were built with alternating layers of poly(dimethyldiallylammonium chloride) (PDDA) and SWNTs. The enhanced mechanical properties of SWNT thin films can be utilized in a variety of applications. To investigate the potential applications of SWNT thin films, magnetic cantilever arrays composed of PDDA, SWNTs, and $\mathrm{Fe}_{2} \mathrm{O}_{3}$ nanoparticles were fabricated.

\section{MATERIALS}

SWNTs (1.1 $\mathrm{nm}$ in diameter, $50 \mu \mathrm{m}$ in length) were purchased from Chengdu Organic Chemicals Co. Ltd. The polyelectrolytes, including PDDA (polycation, molecular weight MW 200,000 to $350,000,3 \mathrm{mg} / \mathrm{ml}, 0.5 \mathrm{M} \mathrm{NaCl}$ ) and poly(sodium 4-styrenesulfonate) (PSS) (polyanion, MW 70,000, $3 \mathrm{mg} / \mathrm{ml}, 0.5$ $\mathrm{M} \mathrm{NaCl}$ ) were obtained from Sigma-Aldrich. Magnetic iron oxide nanoparticles $\left(\mathrm{Fe}_{2} \mathrm{O}_{3}, 50 \mathrm{~nm}\right.$ in diameter, surface covered with hydrophilic PSS, concentration: $2 \mathrm{mg} / \mathrm{ml}$ ) were obtained from Chemicell $\mathrm{GmbH}$. The $\mathrm{Fe}_{2} \mathrm{O}_{3}$ nanoparticles are negatively charged due to the surface coverage of PSS. All materials were diluted in deionized (DI) water.
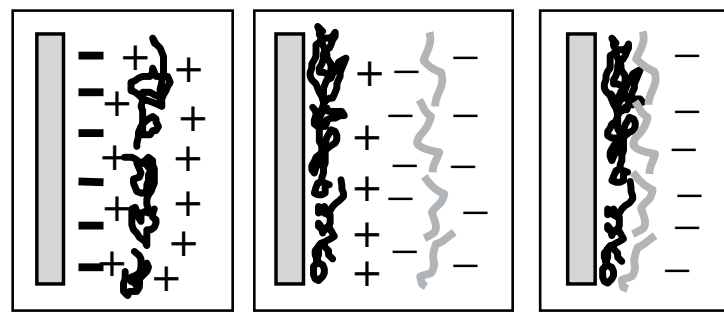

(a) $\{\text { Polycation/SWNTs }\}_{\mathrm{m}}$
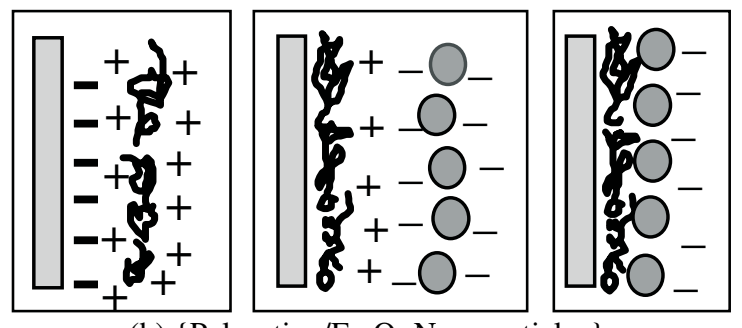

(b) $\left\{\text { Polycation } / \mathrm{Fe}_{2} \mathrm{O}_{3} \text { Nanoparticles }\right\}_{\mathrm{n}}$

Figure 1. Schemes of layer-by-layer alternate assembly of (a) polycation/SWNTs and (b) polycation/ $/ \mathrm{Fe}_{2} \mathrm{O}_{3}$ nanoparticles.

It is well known that pristine SWNTs suffer from poor solubility in water and other chemicals. The hybrid polymer/SWNT composites often have problems such as poor polymer-SWNT connectivity, phase segregation, and structural inhomogeneities [10]. LbL self-assembly is a good approach to 
overcome these problems because it is a solution-based technique. SWNTs with rational treatment can be dispersed in DI water and uniformly deposited on the substrate. Highly homogenous polymer/SWNT thin films with enhanced mechanical properties can be fabricated. The schemes of self-assembled thin films through alternate adsorption of oppositely charged polyelectrolytes, SWNTs, and $\mathrm{Fe}_{2} \mathrm{O}_{3}$ nanoparticles are shown in Fig. 1.

To increase the solubility in DI water, the SWNTs were chemically functionalized by a mixture of nitric and sulfuric acid $\left(1: 3 \mathrm{HNO}_{3}: \mathrm{H}_{2} \mathrm{SO}_{4}\right)$ at $110^{\circ} \mathrm{C}$ for $45 \mathrm{~min}$. The mixture of acids cut the SWNTs into short tubes with openings at both ends. Carboxylic (-COOH) functional groups were covalently attached to the open ends and sidewalls. Next, the SWNT dispersion was diluted with DI water and filtered with a PVDF membrane (with pore diameter of $0.22 \mu \mathrm{m}$ ). The SWNTs were then rinsed with DI water to remove the residual acids. The treated SWNTs were dispersed in DI water. The SWNT dispersion was ultrasonically vibrated for 1 hour. The final step was to remove the excessive SWNTs by centrifuging the SWNT dispersion at $5000 \mathrm{rpm}$ for 15 min. After the chemical funcationalization, the SWNTs were negatively charged and uniformly dispersed in DI water with a concentration of $1 \mathrm{mg} / \mathrm{ml}$.

\section{SWNT MICROPATTERNS}

In previous reports, we presented the patterning of nanoparticle-based structures with the technique that combines the LbL self-assembly, lithography, and lift-off [12]. The same approach can be used to pattern SWNT thin films, as shown in Fig. 2. We have successfully fabricated micro-scale patterns on both silicon and polymer substrates.

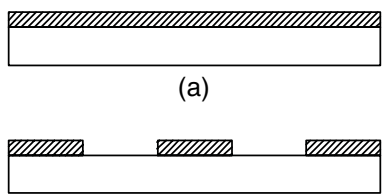

(b)

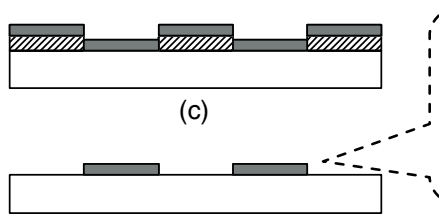

(d)

\section{Substrate}

Photoresist

SWNT thin film

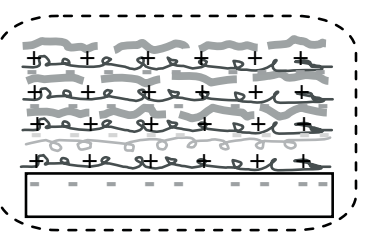

(e)
Figure 2. Fabrication process of the SWNT micropatterns by LbL self-assembly, lithography, and lift-off.

The patterning of the SWNT thin films starts by depositing a $1.5 \mu \mathrm{m}$ layer of photoresist (PR1813) on a substrate (Fig. 2a). Conventional lithography is used to pattern the photoresist layer (Fig. 2b). The next step is to deposit the SWNT multilayer with LbL self-assembly (Fig. 2c). The substrate is soaked into different solutions, and the sequence of the immersion is: [PDDA $(10 \mathrm{~min})+\operatorname{PSS}(10 \mathrm{~min})]_{2}+[\operatorname{PDDA}(10 \mathrm{~min})+$ SWNTs $(15$ $\min )]_{n}$, where $n$ represents the number of (PDDA/SWNT) bilayers. The coating of the first two (PDDA/PSS) bi-layers is to prepare a smooth base for the subsequent coating. Intermediate rinsing with DI water and drying with a nitrogen flow are required to remove the excessive polyelectrolytes and SWNTs. Figure 1e shows the detailed construction of the SWNT multilayer. The final step is to dissolve the photoresist and remove the SWNT multilayer with the lift-off technique (Fig. 1d). The substrate is soaked in acetone for $1 \mathrm{~min}$ with the assistance of ultrasonic vibration. The self-assembled SWNT multilayer is permeable for acetone. Acetone molecules penetrated through the SWNT thin film can dissolve the photoresist and strip off the SWNT multilayer in those areas. Only the SWNT multilayer directly assembled on the substrate remains.

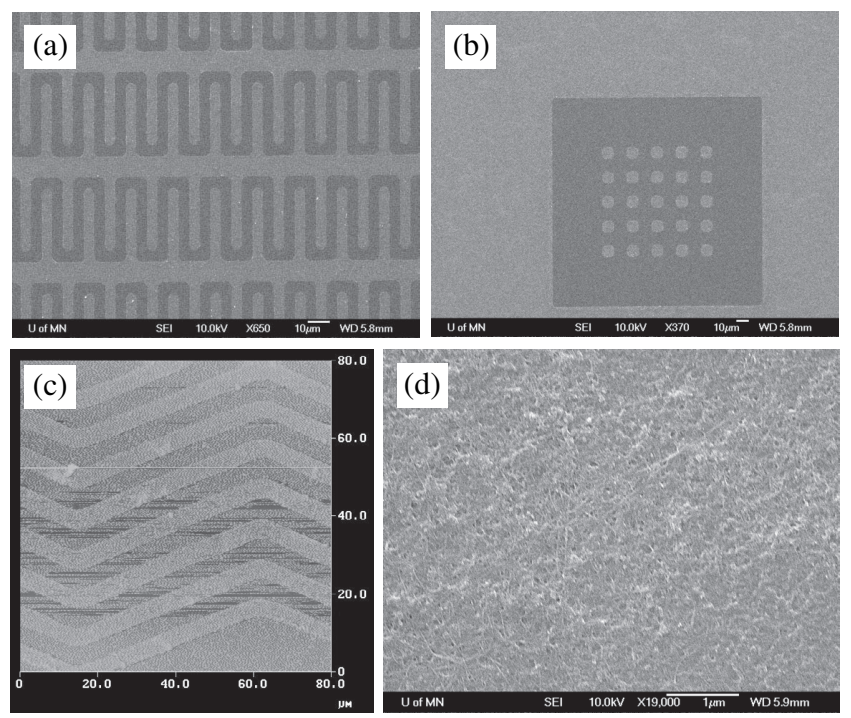

Figure 3. Images of $(P D D A / S W N T)_{6}$ micropatterns. SEM images of (a) micro-springs with linewidth of $5 \mu \mathrm{m}$ and $(b)$ micro-squares with dimensions of $10 \mu \mathrm{m} \times 10 \mu \mathrm{m}$. (c) AFM image of microsprings with linewidth of $5 \mu \mathrm{m}$. The scanning area is $80 \mu \mathrm{m} \times 80$ um. (d) Close inspection of the assembled SWNTs on the substrate with SEM.

Scanning electron microscopy (SEM, JEOL 6500) and atomic force microscopy (AFM, Digital Instruments Dimension 3000, tapping mode) were used to inspect the SWNT micropatterns created with the fabrication technique described above. Figure 3 shows the SEM and AFM images of SWNT micropatterns and self-assembled SWNTs on the substrate. The samples used for microscopy inspection are (PDDA/SWNT) 6 micropatterns. The SWNT thin film is formed by a dense network of SWNTs. The SWNTs are randomly deposited on the surface. The length of the SWNTs is in the range of $1-2 \mu \mathrm{m}$ which is due to the chemical treatment. However, as shown in the figure, the diameters of most SWNTs are much larger than pristine SWNTs $(1.1 \mathrm{~nm})$. The reason for this discrepancy is that during the self-assembly process, several SWNTs are wrapped together due to their extremely high aspect ratios. Therefore, the assembled SWNTs are mainly in the form of nanotube bundles which are approximately $5-10 \mathrm{~nm}$ in diameter and $1-2 \mu \mathrm{m}$ in length.

To estimate the thickness of the SWNT multilayer, a group of $50 \mu \mathrm{m}$ lines composed of (PDDA/SWNT) 6 multilayer was fabricated on a silicon substrate using the method described above. Surface profiler (Dektak, Model IIA) was used to measure the vertical profile of the SWNT lines. The average thickness of the (PDDA/SWNT) 6 structure was measured approximately as $400 \AA$. The surface profiler obtains its measurement data by moving a diamond tip stylus over the patterns during the scan. It usually gives relatively accurate results on hard substrates. However, when used on soft thin films, the scanning is destructive and may give inaccurate results. In order to verify the measured results from the surface profiler, an ellipsometer 
(Gaertner Scientific Co.) was used to detect the thin film thickness. Ellipsometer provides a non-destructive method and gives more accurate results once the rough thickness of the film is known. The measured thickness of the (PDDA/SWNT) 6 multilayer was $455.4 \pm 5 \% \AA$. Therefore, the average thickness of a (PDDA/SWNT) bi-layer is approximately $76 \AA$.

\section{SWNT-BASED MAGNETIC CANTILEVER ARRAY}

The mechanical properties of the LbL self-assembled SWNT thin film were studied and reported. The Young's modulus of the SWNT film was measured as $17-35 \mathrm{GPa}$, and the ultimate tensile strength was measured as $180-325 \mathrm{MPa}$ [10]. Compared with strong polymers which are commonly used for MEMS devices such as polymethyl methacrylate (PMMA, Young's modulus: $2 \mathrm{GPa}$, ultimate tensile strength: $50 \mathrm{MPa}$ ), SWNT thin films are several times stronger. SWNT films also have enough flexibility due to its composite essence. In general, the SWNT thin films have the mechanical properties between those of silicon and polymers. The SWNT thin films can serve as structural components in MEMS devices to provide both flexibility and strength. In addition, the "bottom-up" LbL self-assembly technique has the ability to control the film thickness in nanometer scale. Therefore, the SWNT cantilever array may have the potential to be used in a variety of applications.
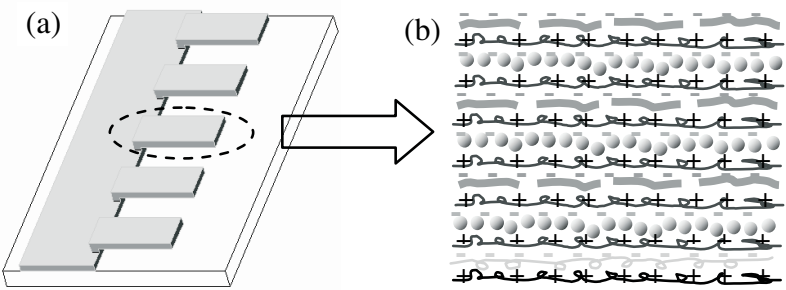

Figure 4. (a) Scheme diagram of the cantilever array fabricated on silicon substrate. (b)Illustration of the SWNT-based magnetic cantilever multilayer. The multilayer consists of PDDA (+), PSS (-), $\mathrm{Fe}_{2} \mathrm{O}_{3}$ nanoparticles (-), and SWNTs (-).

To investigate the potential applications of SWNT thin films, especially in biosensing, SWNT-based magnetic cantilever arrays were designed and fabricated. The schematic diagram of the SWNT cantilever array is illustrated in Fig. 4. Both the roots and the beams are composed of SWNT multilayer. Iron oxide nanoparticles are integrated in the multilayer as magnetically sensitive material, which makes the measurement convenient. The cantilever array can be used in detecting biomolecules such as proteins, enzymes, and DNAs. A number of biomolecules are naturally charged and can be assembled in alternation with polyelectrolytes based on LbL self-assembly technique. Because the cantilevers are self-assembled multilayers, the surfaces of the cantilevers are charged. The biomolecules can be readily adsorbed onto the cantilevers. The resonant frequency shift caused by the mass increase of the cantilever can be detected by an optical interferometer. Another possible application is to use the cantilever beams as magnetically driven microvalves.

The fabrication process of the cantilever array is shown in Fig. 5. A layer of photoresist PR1813 is spin coated on the substrate (Fig. 5a). A window for the cantilever root is opened using ultraviolet (UV) lithograph (Fig. 5b). Second UV lithography is executed with part of the PR1813 layer protected. The protected PR1813 is used as the sacrificial layer which is underneath the cantilever beam (Fig. 5c). The
PDDA/PSS/SWNT/ $/ \mathrm{Fe}_{2} \mathrm{O}_{3}$ multilayer is self-assembled on the substrate (Fig. 5d). The immersion sequence is [PDDA (10 min) + PSS $(10$ min $)]_{2}+\left\{\right.$ PDDA $(10 \mathrm{~min})+\mathrm{Fe}_{2} \mathrm{O}_{3}(15 \mathrm{~min})+[$ PDDA $(10$ min $)+$ SWNTs $(15$ min $)]_{2}+$ PDDA $(10$ min $)+\mathrm{Fe}_{2} \mathrm{O}_{3}(15$ $\min )+\operatorname{PDDA}(10 \mathrm{~min})+\operatorname{SWNTs}(15 \mathrm{~min})\}_{3}$. Nine layers of SWNTs are used to strengthen the cantilever structure. Six layers of $\mathrm{Fe}_{2} \mathrm{O}_{3}$ nanoparticles make the cantilever magnetically sensitive. The growth of the multilayer was monitored by a quartz crystal microbalance (QCM). The effective surface coverage of the $\mathrm{Fe}_{2} \mathrm{O}_{3}$ nanoparticles was measured approximately as $30 \%$. The effective thickness of the multilayer was calculated approximately as $200 \mathrm{~nm}$. The exposed PR1813 is dissolved by 351 developer with the lift-off technique. In order to remove the multilayer coated on the exposed PR1813, the lift-off process is implemented with ultrasonic vibration (Fig. 5e). The freestanding cantilever beam is released by stripping off the PR1813 sacrificial layer with acetone (Fig. 5f).

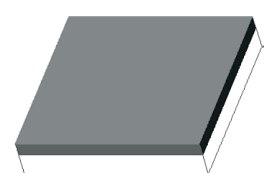

(a)

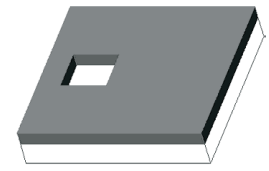

(b)

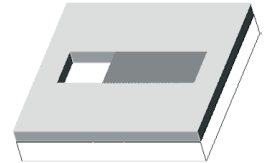

(c)

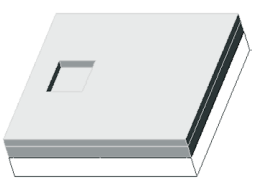

(d)

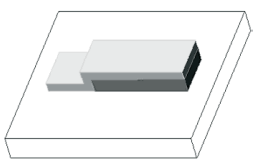

(e)

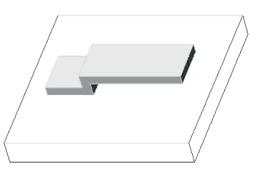

(f)
Figure 5. Fabrication process of the self-assembled cantilever.

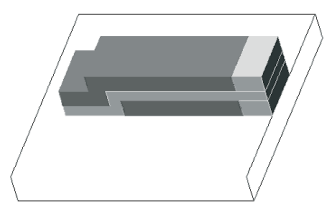

(a)

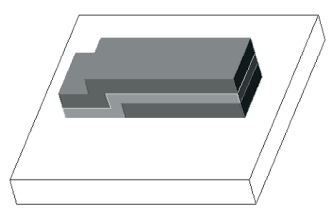

(b)
Figure 6. Modified lift-off process with a "sandwich" structure. (a) Before lift-off. (b) After lift-off with 351 developer.

The micropatterns fabricated with conventional lift-off processes usually have step profiles. The stripper molecules can easily attack the underlying photoresist layer and remove the unwanted top layer. However, as shown in Fig. 5d, the selfassembled multilayer covers the whole surface of the device and is almost at the same height. During the lift-off process, ultrasonic vibration is required to break the multilayer. It takes 8 $-10 \mathrm{~min}$ to remove the unwanted parts of the thin film due to its high mechanical strength. The long-time ultrasonic bath severely weakens and damages the cantilever structures, especially the joints between the roots and the beams. The weakening of the structures may cause detachment of the beams from the roots. Therefore, a modified lift-off process was developed to replace the lift-off step shown in Fig. 5e. A "sandwich" structure provides additional protection for the cantilever roots and beams. As shown in Fig. 6, a top photoresist layer is coated to cover the surface of the multilayer. It should be noted that only part of the 
multilayer is shown in the figure for visibility. The whole surface is actually covered by photoresist. The parts of the photoresist that cover the cantilever roots and beams are protected by photomask while other parts of the photoresist are exposed under UV light. Based on the modified lift-off process, the ultrasonic bath time can be greatly reduced to $2 \mathrm{~min}$. Both the cantilever roots and beams are well protected by the top photoresist layer.

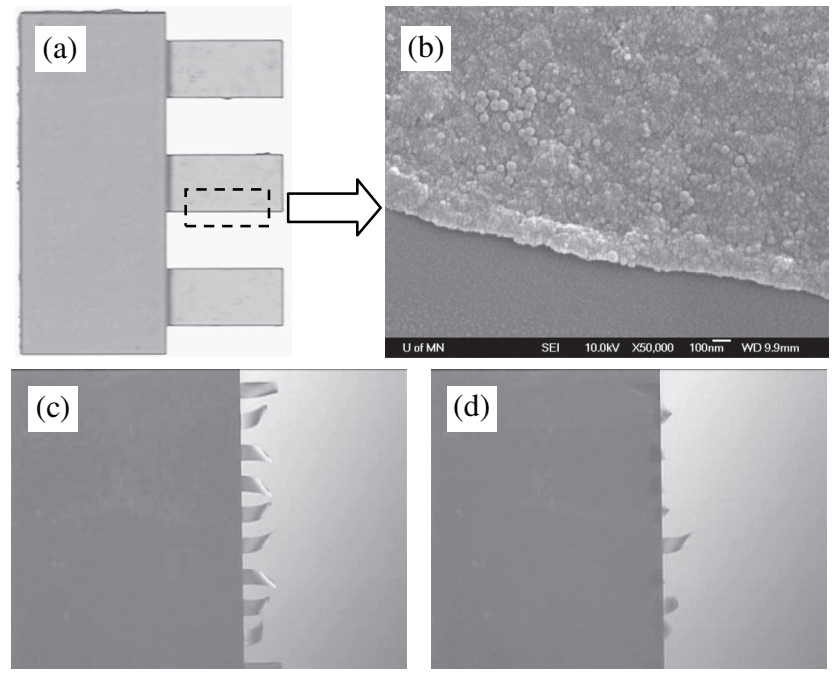

Figure 7. Images of the LbL self-assembled cantilever array. (a) Optical image of the cantilever array. The length and the width of the beams are $300 \mu \mathrm{m}$ and $150 \mu \mathrm{m}$, respectively. (b) SEM inspection of the edge of the cantilever. (c) Cantilever array in acetone solution without external magnetic field. (d) Flexed cantilever array under the influence of external magnetic field. The beams are $150 \mu \mathrm{m}$ in length and $50 \mu \mathrm{m}$ in width.

The images of the fabricated cantilever array are shown in Fig. 7. Figure 7a shows an optical image of a cantilever array consists of three beams. Figure $7 \mathrm{~b}$ shows the SEM inspection of the edge of the beam. The $\mathrm{Fe}_{2} \mathrm{O}_{3}$ nanoparticles can be clearly seen from the figure. To strip off the top protecting and the bottom sacrificial photoresist layers, the device is submerged in acetone. The beams float in the solution when there is no external magnetic field (Fig. 7c). When an external magnetic field is applied by holding a permanent magnet $1 \mathrm{~cm}$ above the device, the beams are deflected (Fig. 7d). The deflection angles can exceed 90 degrees in the presence of the magnetic field. The beams restore rapidly after moving the magnet away from the device. There is no observable structural failure or damage after repeated deflections (more than 100 times). The SWNT multilayer is proven to be very flexible and strong. However, the internal stress inside the SWNT multilayer is not neglectable. The free ends of the cantilever beams are curled in the acetone solution. This may cause measurement problems when optical method is used. Further studies will be conducted to solve the problem. Possible solutions include increasing the multilayer thickness, trimming the corners of the beams, increasing the number of SWNT layers, etc.

\section{CONCLUSIONS}

We have presented an effective, simple, low-cost, and lowtemperature approach to fabricate SWNT thin films and micropatterns using LbL nano self-assembly and UV lithography. SWNT micropatterns with features as small as $5 \mu \mathrm{m}$ have been produced. It is believed that the patterned feature size can be downscaled to submicron or even nanoscale if high-resolution lithography techniques such as electron-beam lithography are used. The assembled SWNTs are mainly in the form of nanotube bundles which are approximately $5-10 \mathrm{~nm}$ in diameter and $1-2$ $\mu \mathrm{m}$ in length. The thickness of a (PDDA/SWNT) bi-layer is approximately $76 \AA$. LbL self-assembled cantilever array composed of polyions/SWNTs/nanoparticles multilayer has been fabricated. A modified lift-off process provides additional protection for the cantilever structures. The strength and thickness of the cantilever can be adjusted in a wide range. The cantilever beams can be easily deflected to over $90^{\circ}$. The fabricated cantilever array can be used as magnetically driven microvalves. It can also be used in biosensing applications for biomolecules adsorption and detection because a number of biomolecules can be easily adsorbed on the self-assembled multilayer.

\section{REFERENCES}

[1] S. Iijima, "Helical Microtubules of Graphitic Carbon", Nature, 354, 56 (1991).

[2] J. Kong, N.R. Franklin, C. Zhou, M.G. Chapline, S. Peng, K. Cho, H. Dai, "Nanotube Molecular Wires as Chemical Sensors", Science, 287, 622 (2000).

[3] K.A. Williams, P.T.M. Veenhuizen, B.G. de la Torre, R. Eritja, C. Dekker, "Carbon Nanotubes with DNA recognition", Nature, 420, 761 (2002).

[4] W.B. Choi, D.S. Chung, J.H. Kang, H.Y. Kim, Y.W. Jin, I.T. Han, Y.H. Lee, J.E. Jung, N.S. Lee, G.S. Park, J.M. Kim, "Fully Sealed, High-Brightness Carbon-Nanotube Field-Emission Display", Applied Physics Letters, 75, 3129 (1999).

[5] M. Penza, F. Antolini, M.V. Antisari, "Carbon Nanotubes as SAW Chemical Sensors Materials", Sensors and Actuators, B: Chemical, 100, 47 (2004).

[6] J. Kong, M. G. Chapline, H. Dai, "Functionalized Carbon Nanotubes for Molecular Hydrogen Sensors", Advanced Materials, 13, 1384 (2001).

[7] L. Valentini, C. Catalini, I. Armentano, J.M. Kenny, L. Lozzi, S. Santucci, "Highly Sensitive and Selective Sensors Based on Carbon Nanotubes Thin Films for Molecular Detection", Diamond and Related Materials, 13, 1301 (2004).

[8] Y. Jang, S. Moon, J. Ahn, Y. Lee, B. Ju, "A Simple Approach in Fabricating Chemical Sensor using Laterally Grown MultiWalled Carbon Nanotubes", Sensors and Actuators, B: Chemical, 99, 118 (2004).

[9] Younan Xia, George M. Whitesides, "Soft Lithography", Annual Review of Materials Science, 28, 153, (1998).

[10 A.A. Mamedov, N.A. Kotov, M. Prato, D.M. Guldi, J.P. Wicksted, A. Hirsch, "Molecular Design of Strong Single-Wall Carbon Nanotube/Polyelectrolyte Multilayer Composites", Nature Materials, 1, 190 (2001).

[11] M. Zhang, K. Gong, H. Zhang, L. Mao, "Layer-by-Layer Assembled Carbon Nanotubes for Selective Determine of Dopamine in the Presence of Ascorbic Acid“, Biosensors and Bioelectronics, 20, 1270 (2005).

[12] F. Hua, T. Cui, Y. Lvov, "Ultrathin cantilevers based on polymer-ceramic nanocomposite assembled through layer-by-layer adsorption", Nano Letters, 4, 823, (2004). 